\title{
10. Mainzer Workshop - Wolfgang Schmutzler gewidmet
}

Z um 10. Mal fand vom 6. bis 7 . März 1998 der Mainzer Workshop der DGAI statt, auf dem junge Allergieforscher in Klinik und Labor ihre wissenschaftlichen Ergebnisse vorstellen. Diese 10jährige Entwicklung ist Anlaß zur ungetrübten Freude: Eine junge Generation von Forschern und klinischen Allergologen ist an verschiedensten Orten, in unterschiedlich großen Arbeitsgruppen und von verschiedensten Disziplinen herkommend, herangewachsen. Es ist wichtig, daß wir Allergologen die Neuentwicklungen der Grundlagendisziplinen verstehen und in unsere Forschung einbeziehen: Grundlagendisziplinen wie Genetik, Zellbiologie, Biochemie, Biologie, Pharmakologie und Immunologie, ebenso aber auch Epidemiologie, Psychosomatik, Public Health und viele andere. Genauso wichtig ist die gute allergologische Klinik, die für die Zukunft unserer Disziplin entscheidend ist.

Klinische Forschung ist nicht einfach Materiallieferung, ist aber auch nicht Grundlagenforschung in der Klinik; Grundlagenforschung gehört in Spezialinstitute. Klinische Forschung ist Forschung am und für den Patienten. Diese wissenschaftlich genauso hochstehende und anstrengende Arbeit wird in der heutigen Zeit oft unterschätzt, die Identifikation, Auswahl, Dokumentation und Klassifikation der Krankheitsbilder für wissenschaftliche Untersuchungen für selbstverständlich gehalten.

Die Fragen der Forschung müssen vom Patienten und der klinischen Situation herkommen. Forschung muß echte Probleme lösen und wirkliche Fragen beantworten. Viel zu oft wird heute nach dem Motto geforscht ,da ist eine teure Maschine, die lassen wir einmal über verschiedene Diagnosen laufen“ oder ,das hat noch keiner gemacht" oder - bei den besonders Erfolgreichen - „das lesen die Editoren von High-Impact-Journals gerne“.
Am Anfang muß eine Frage stehen, die mit wissenschaftlichen Methoden beantwortet werden soll und kann und die letztlich dem Patienten hilft. Alles andere ist Tand, auch wenn es kurzzeitig auf der Woge bestimmter wissenschaftlicher Modeströmungen hoch dekoriert wird.

Wenn die Allergologie in Deutschland überleben soll, müssen die Allergologen die Patienten besser behandeln als andere Ärzte.

Und wir müssen den Gedanken der Interdisziplinarität unseres Faches hochhalten; das befreit uns von dem Makel ausschließlicher Interessenvertretung, die wir außerhalb unserer Gemeinde nicht vermitteln können.

Deshalb hat die DGAI auch auf dem letzten Kongreß Sektionen gegründet, die einerseits die relevanten Disziplinen einbinden und andererseits in dieselbe allergologische Expertise ausstrahlen sollen. Das sind folgende Sektionen und Sprecher: Dermatologie (Kapp, Hannover), Hals-Nasen-Ohren-Heilkunde (Bachert, Gent), Pneumologie (Bergmann, Bad Lippspringe), Pädiatrie (Bauer, München), Umweltmedizin (Behrendt, Hamburg) und Immunologie (Renz, Berlin). Wer interessiert ist mitzuarbeiten, möge sich bei den Sprechern melden. Auch die Arbeitsgruppen der DGAI sind tätig, besonders die der „Nahrungsmittelallergie“ (Jäger, Jena), die soeben ein Positionspapier vorgelegt hat, und die Arbeitsgruppe „Allergenkarenz“ (Müsken, Bad Lippspringe), die die Kriterien für sinnvolle Allergenkarenzmaßnahmen festlegen soll.

Warum hat die DGAI den 10. Mainzer Workshop Herrn Prof. Dr. Wolf-

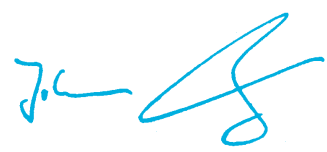

Prof. Dr. Dr. J. Ring gang Schmutzler gewidmet? Wolfgang Schmutzler hat vor 10 Jahren diese Tradition ins Leben gerufen und seither ständig mit viel Enthusiasmus und auch kritischen Kommentaren fruchtbar begleitet. Wolfgang Schmutzler scheidet im Frühjahr 1998 aus dem aktiven Universitätsleben aus. Er hat sich als langjähriges Vorstands- und Beiratsmitglied der DGAI, ehemaliger Präsident der DGAI und Organisator des letzten gemeinsamen Kongresses mit der Gesellschaft für Immunologie bleibende Verdienste um die Allergologie in Deutschland erworben.

Wolfgang Schmutzler ist als Pharmakologe auch immer so etwas wie das „Gewissen unserer Gesellschaft" gewesen, ein stetiger Mahner, trotz aller finanziellen Engpässe auf keinen Fall die Seele zu verkaufen! Die Auswahl von Themen und Rednern auf den Tagungen der Allergologie muß ausschließlich nach wissenschaftlichen und klinischen Gesichtspunkten erfolgen; dies verstehen auch professionelle Sponsoren, die nur von der echten Botschaft langfristige Erfolge erwarten können.

An dieser Stelle kann keine Laudatio für Wolfgang Schmutzler stehen. Wir möchten unsere Leser auf die Laudatio im Allergo Journal 8/1996 anläßlich der Verleihung der KarlHansen-Medaille verweisen.

DGAI und Allergo Journal wünschen Wolfgang Schmutzler alles Gute zum 65. Geburtstag; vor allem wünschen wir, daß der Deutschen Allergologie sein ehrliches Bemühen, sein allzeit kritischer und gleichzeitig menschenfreundlicher und offener Geist noch lange Jahre erhalten bleibt!

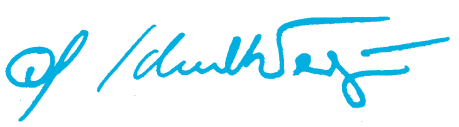

Prof. Dr. G. Schultze-Werninghaus 